\title{
Joint replacement surgery in Ghana (West Africa)
}

\author{
Zuyun Yan ${ }^{1} \cdot$ Xinqiao Tang ${ }^{1} \cdot$ Xiaoming Chen $^{1} \cdot$ Zhong Liu $^{1}$
}

Received: 16 February 2019 / Accepted: 25 February 2019 / Published online: 20 March 2019

(C) SICOT aisbl 2019

Dear Editor,

In the article entitled "Joint replacement surgery in Ghana (West Africa)-an observational study," Akintunde George and Paul Ofori-Atta performed a prospectively study to summarize hip and knee arthroplasty work of an orthopaedic charity in a developing country over a period of four years [1]. It is a valuable study, nevertheless, there are some queries that we would like to communicate with the authors.

1. In this study, the average age of patients who received THA was 47.4 years, which is younger than that of developed countries like the UK or Norway. However, as stated by the authors, the patients in Ghana were able to tolerate pain due to financial constrain. This creates discrepancies; the average age should be older rather than younger. We speculate this may be explained by the high percentage of osteonecrosis of the femoral head (ONFH) population in this study. According to NJR 15th Annual Report, osteoarthritis (OA) is the sole reason for THA for $88.8 \%$ patients [2]. But in this study, only $52 \%$ patients received THA because of OA, and a high population of $23 \%$ is diagnosed with ONFH. The patients of ONFH are of younger age, and is it possible that the high ratio of this patients contributes to the younger THA population? Moreover, is the incidence rate of ONFH higher in Ghana than other countries? And if yes, what might be the underlying reasons for this?

2. The youngest patients receiving THA in this study is a 17-year-old patient. It is uncommon for an adolescent to receive joint arthroplasty, what are pre-operative conditions of this patient? The author had documented the basic diagnostic information of patients, but no specific data are given in the paper, it would be wonderful if these detailed pre and postoperative information of patients could be given.

Zhong Liu

liuzhwangf@163.com

1 Department of Orthopedics, Xiangtan Central Hospital, Clinical Practice, Base of Central South University, 120 Heping Road, Xiangtan 411100, Hunan, China
3. In the study, the patellofemoral arthroplasty rate is $28 \%$, which is relatively lower than that of Portugal, which is $66 \%$. What are the underlying reasons for this phenomenon? As no detailed information of the patient enrollment criteria are given in the paper, could this be explained by the patients' tolerance of pain and their economic conditions? Moreover, as the ideal candidates for patella replacement are patients with trochlear dysplasia or isolated patellofemoral osteoarthritis, is there a lower incident rate of these conditions in Ghana that contributes to lower patellofemoral arthroplasty rate?

4. In the study, for the hip arthroplasty, $72.6 \%$ them were uncemented, $18.6 \%$ were cemented, and $8.8 \%$ were hybrid. The uncemented prosthesis used in Ghana was more than those used in the British experience, which is $40 \%$ [3]. It may be partially explained by the younger age, but are there other factors that contribute to this phenomenon? Is this study a single centre study, or can it represent the whole hip arthroplasty conditions in Ghana? Is it because of the relatively smaller patient number of this study? Or is it because of the surgeons' preferences, and what are their considerations when choosing candidates to receive uncemented prosthesis or cemented prosthesis?

\section{Compliance with ethical standards}

Conflict of interest The authors declare that they have no conflict of interest.

\section{References}

1. George A, Ofori-Atta P (2019) Joint replacement surgery in Ghana (West Africa)-an observational study. Int Orthop. https://doi.org/10. 1007/s00264-019-04286-1

2. (Chairman) LP-F (2018) 15th Annual Report 2018, National Joint Registry for England, Wales, Northern Ireland and the Isle of Man. NJR Report website.

3. Porter M, Whitehouse M (2017) What does the NJR's 14th Annu Rep tell us. In: British Orthopaedic Association Annual Conference, Liverpool, 2017

Publisher's note Springer Nature remains neutral with regard to jurisdictional claims in published maps and institutional affiliations. 